DOI: $10.24850 / j-$ tyca-2022-01-01

Artículos

\title{
Estudio numérico-experimental del campo de velocidades en un canal rectangular con curvatura compuesta y ancho variable en 2DH
}

\section{Experimental-numerical study of the velocity field in a rectangular channel with composite curvature and variable width in $\mathbf{2 D H}$}

José Luis Aragón-Hernández ${ }^{1}$, ORCID: https://orcid.org/0000-00015102-1598

Christian Alberto Caballero-Coranguez², ORCID: https://orcid.org/00000001-9080-7083

Amado Abel Jiménez-Castañeda3, ORCID: https://orcid.org/0000-00032081-5429

Moisés Berezowsky-Verduzco4, ORCID: https://orcid.org/0000-0002$7675-3450$

${ }^{1}$ Departamento de Hidráulica, División de Ingenierías Civil y Geomática, Facultad de Ingeniería, Universidad Nacional Autónoma de México, Ciudad de México, México, jaragonh@unam.mx 
${ }^{2}$ Coordinación de Hidráulica, Subdirección de Hidráulica y Ambiental, Instituto de Ingeniería, Universidad Nacional Autónoma de México, Ciudad de México, México, ccaballeroc@iingen.unam.mx

${ }^{3}$ Coordinación de Hidráulica, Subdirección de Hidráulica y Ambiental, Instituto de Ingeniería, Universidad Nacional Autónoma de México, Ciudad de México, México, ajimenezc@iingen.unam.mx

${ }^{4}$ Coordinación de Hidráulica, Subdirección de Hidráulica y Ambiental, Instituto de Ingeniería, Universidad Nacional Autónoma de México, Ciudad de México, México, mbv@pumas.ii.unam.mx

Autor para correspondencia: José Luis Aragón-Hernández, jaragonh@unam.mx

\section{Resumen}

Se presenta un análisis de la aproximación obtenida con los sistemas de modelación matemática HEC-RAS, Iber y Telemac-Mascaret para el cálculo del campo de velocidades en un canal de sección rectangular con curvatura compuesta y ancho variable; en el canal se desarrollaron corrientes secundarias y separación de flujo; los tres modelos son de uso libre y se basan en la hipótesis de flujo bidimensional horizontal (2DH). Se utilizan las ecuaciones de Saint Venant en cada modelo matemático para calcular la hidrodinámica y la turbulencia. El campo de velocidades se determina a partir de mediciones físicas en un canal de laboratorio con un velocímetro Doppler acústico (Micro-ADV $16 \mathrm{MHz}$ ) y se compara con 
los campos de velocidades calculados con los sistemas de modelación matemática (HEC-RAS, Iber y Telemac-Mascaret). Se presenta un análisis de errores, con el objetivo de determinar el grado de aproximación obtenido con cada sistema de modelación, y la discusión de los factores y fenómenos observados en el laboratorio, esto permite tener conocimiento sobre la complejidad en la naturaleza de los flujos turbulentos en canales con curvaturas fuertes. Se encontró que los tres modelos, en general, tienen un comportamiento similary aceptable, con una aproximación promedio superior al $93 \%$; en cambio, en un análisis por sección, en una zona de recirculación, se observó separación del flujo y alta turbulencia (procesos físicos de la dinámica del fluido que los modelos empleados no pueden reproducir de forma adecuada); la aproximación promedio disminuye hasta el $82.5 \%$.

Palabras clave: canal curvo, modelación numérica bidimensional horizontal, hidrodinámica, turbulencia, separación de flujo.

\section{Abstract}

An analysis of the approximation obtained with the mathematical modeling systems HEC-RAS, Iber, and Telemac-Mascaret is presented, for calculation of velocity field in a rectangular section channel with compound curvature and variable width. On the channel, secondary currents, and flow separation developed; all three models are free to use and based on the horizontal two-dimensional flow (2DH) hypothesis. The Saint Venant equations are used in each mathematical model to calculate 
hydrodynamics and turbulence. The velocity field is determined from physical measurements in a laboratory channel with an acoustic Doppler velocimeter (Micro-ADV $16 \mathrm{MHz}$ ) and it is compared with the velocity fields calculated with the mathematical modeling systems (HEC-RAS, Iber, and Telemac-Mascaret). An error analysis is presented, to determine the degree of approximation obtained with each modeling system and discussion of the factors and phenomena observed in the laboratory, this allows knowing the complexity like turbulent flows in channels with strong bent. It was found that the three models, in general, have similar and acceptable behavior, with an average approximation greater than $93 \%$; on the other hand, in an analysis by section, in a recirculation zone, flow separation and high turbulence were observed (physical processes of fluid dynamics that the models used cannot reproduce adequately); the average approximation decreases to $82.5 \%$.

Keywords: Bend channel, horizontal two-dimensional numerical modeling, hydrodynamics, turbulence, flow separation.

Recibido: $24 / 08 / 2020$

Aceptado: 02/12/2020 
Tecnología y

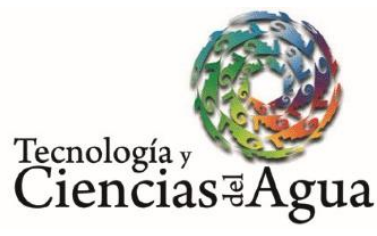

2022, Instituto Mexicano de Tecnología del Agua

Open Access bajo la licencia CC BY-NC-SA 4.0

(https://creativecommons.org/licenses/by-nc-sa/4.0/)

\section{Introducción}

En Jiménez y Berezowsky (2004) se presentó el desarrollo de un sistema de modelación matemática para calcular el campo de velocidades en canales, cuyas fronteras laterales tienen una geometría irregular o con curvas en planta; consideraron coordenadas curvilíneas generales para adaptarse a estas geometrías y usaron la versión covariante con componentes físicos de las ecuaciones de hidrodinámica para aproximar la solución a las ecuaciones promediadas en la profundidad, considerando la hipótesis de flujo bidimensional horizontal; las ecuaciones diferenciales las resolvieron en diferencias finitas con el esquema de MacCormack (Garcia \& Kahawita, 1986; Fennema \& Chaudhry, 1990), que es del tipo explícito y de segundo orden; incluyeron el cálculo de la viscosidad turbulenta $\left(v_{t}\right)$ con los modelos parabólico y $k-\varepsilon$. El trabajo contiene cinco geometrías distintas sobre las cuales se valida el modelo matemático desarrollado; presentan mediciones experimentales y velocidades calculadas; muestran el proceso de calibración, verificación y validación. Los autores indican que la comparación es similar en la mayoría de los casos; sin embargo, en una de las aplicaciones existen diferencias apreciables entre los campos de velocidades medidos y calculados en una pequeña zona, en principio asociadas con limitaciones en el tamaño de la malla de cálculo, aunque no presentan una explicación 
que permitiera comprender esta variación entre la velocidad medida y calculada.

Luo (2013) presentó un estudio referido a la separación del flujo y a la recirculación que se tienen en las expansiones de canales; describe el cálculo de los perfiles de velocidad, los perfiles de esfuerzos cortantes $(\tau)$ debidos a la turbulencia, los perfiles de energía cinética turbulenta $(k)$, así como la tasa de disipación de energía y el coeficiente de dispersión. Los resultados medidos fueron comparados con los calculados al emplear un modelo de hidrodinámica que utiliza las ecuaciones promediadas en la profundidad y cinco modelos de turbulencia para el cálculo de la viscosidad turbulenta $\left(v_{t}\right)$. Un trabajo pionero y fundamental sobre el análisis de la hidrodinámica en canales con curvas es el desarrollado por Rozovskii (1957).

Con el avance del estado del conocimiento en métodos numéricos y el aumento de la velocidad de los equipos de cómputo, hoy en día las ecuaciones de Saint Venant en dos dimensiones se utilizan para el cálculo de la hidrodinámica (Ghamry \& Steffler, 2005; Luo, 2013), la turbulencia (Luo, 2013; Wu, Wang, \& Chiba, 2004), flujos secundarios (Ghamry \& Steffler, 2005; Song, Seo, \& Kim, 2012), separación de flujo y recirculación (Luo, 2013) en canales curvos, con resultados aceptables. Para el cálculo de la turbulencia, además se incluyen los modelos de turbulencia parabólico, longitud de mezcla modificado, $k-\varepsilon, k-\varepsilon \sin$ equilibrio y RNG $k-\varepsilon$ (Luo, 2013; Wu et al., 2004).

En los trabajos anteriores, la discretización del dominio de estudio se puede realizar con coordenadas rectangulares (Luo, 2013) y 
coordenadas curvilíneas generales (Kalkwijk \& De-Vriend, 1980; Jiménez \& Berezowsky, 2004); en el primer caso conlleva limitaciones en la flexibilidad de la malla y en el cálculo de soluciones con discontinuidades (Bladé et al., 2014b); en cambio, en el segundo permite tener una mejor aproximación en la representación de fronteras con curvatura (Jiménez \& Berezowsky, 2004).

Hoy en día se tienen distintos sistemas de modelación matemática, algunos contienen sus propios generadores de mallas bastante eficientes, con elementos de tres (triángulos), cuatro (cuadriláteros) o más lados que permiten tener una mejor aproximación de las fronteras y refinado de la malla en distintas zonas. Entre estos modelos destacan aquellos que son de uso libre, principalmente HEC-RAS, Iber y Telemac-Mascaret; dichos modelos han sido evaluados y comparados con otros modelos de tipo académico (Horritt et al., 2007; Kaveh et al., 2019; Shustikova, Domeneghetti, Neal, Bates, \& Castellarin, 2019) y comerciales (Pinos \& Timbe, 2019; Rousseau, Biron, \& Van-de-Wiel, 2016); todos ellos implementados en distintos estudios hidráulicos para calcular la hidrodinámica en dispositivos experimentales y modelos físicos (García \& Matamoros, 2019; Hafnaoui \& Debabeche, 2020) con buenos resultados.

El uso de modelos numéricos en ingeniería es una herramienta esencial para el diseño de estructuras hidráulicas, verificación y/o validación de las mismas; permite plantear escenarios para la toma de decisiones, determinar vulnerabilidades y la optimización del funcionamiento hidráulico, entre otros. Sin embargo, es necesario conocer las capacidades y limitaciones que ofrecen, con el objetivo de 
Tecnología y

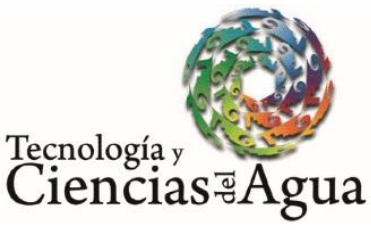

2022, Instituto Mexicano de Tecnología del Agua

Open Access bajo la licencia CCBY-NC-SA 4.0

(https://creativecommons.org/licenses/by-nc-sa/4.0/)

evitar caer en soluciones erróneas; por lo tanto, es fundamental contar con conocimientos de la física del fenómeno y criterios para optar por soluciones óptimas y eficientes (Bladé, Cea, \& Corestein, 2014a). Es así que los modelos numéricos, al ser aproximaciones, deben ser validados y verificados, aunque esto en la aplicación directa en ingeniería en muchas ocasiones resulta complicado de determinar, ya sea porque no se cuenta con mediciones de variables en campo, como lo pueden ser niveles de agua, caudales, velocidades, temperatura, o incluso más complejas como lo es la aceleración, y el transporte de sedimento de fondo y en suspensión (Knight, 2014).

Por otra parte, en los estudios experimentales es muy importante la medición de las variables hidráulicas con dispositivos con resolución temporal y espacial adecuada (Voulgaris \& Trowbridge, 1998). Para ello, se dispone de sofisticados equipos de medición intrusivos y no intrusivos con distintos avances tecnológicos; la mayoría de las investigaciones más recientes implementa técnicas ultrasónicas con equipos intrusivos para medir el campo de velocidades, como los velocímetros Doppler acústicos (ADV), ya sea puntuales o perfiladores en la vertical (Voulgaris \& Trowbridge, 1998; Lane et al., 1998); otras técnicas recientes no intrusivas basadas en la visualización, como la velocimetría por rastreo de partículas (PIV, PTV), están ganado popularidad (Ruonan, Liekai, Xingkui, \& Danxun, 2016). Una muestra de lo anterior es el campo de velocidades medido con un perfilador de velocimetría ultrasónico de efecto Doppler empleado por Bombar, Elçi, Tayfur, Güney y Bor (2011); trabajos similares han sido desarrollados por Da-Silva, El-Tahawy y Tape 
Tecnología y

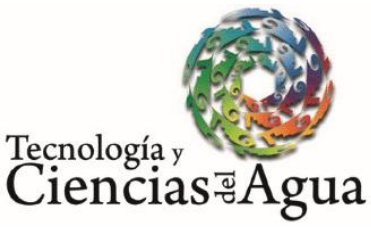

2022, Instituto Mexicano de Tecnología del Agua

Open Access bajo la licencia CC BY-NC-SA 4.0

(https://creativecommons.org/licenses/by-nc-sa/4.0/)

(2006); Abad y Garcia (2009); Termini (2009), y Abad, Frias, Buscaglia y Garcia (2013), entre otros.

El objetivo de este trabajo es presentar el comportamiento hidrodinámico que se desarrolla en un canal con curvas horizontales y ancho variable en planta presentado por Jiménez y Berezowsky (2004), esencialmente el campo de velocidades a partir de mediciones experimentales y el determinado por tres modelos matemáticos: HECRAS, Iber y Telemac-Mascaret. Se presenta la comparación de las velocidades y el análisis del error en cada uno de los modelos empleados. Esto proporciona conocimiento sobre el comportamiento de la hidrodinámica en canales curvos, pues en la mayoría de los casos, las geometrías empleadas son canales de laboratorio rectos sobre los cuales se han validado numerosas aplicaciones, o que presentan un ancho constante a lo largo de una curva, como los trabajos de Abad y Garcia (Abad \& Garcia, 2006; Abad \& Garcia, 2009), pero el uso de estos modelos numéricos en la aplicación en ingeniería de ríos muestra geometrías irregulares más complejas, lo que ocasiona un flujo turbulento, y dificulta la validación de los modelos numéricos con mediciones de campo, e incluso establecer las condiciones en las fronteras para el o los escenarios en estudio.

Una de las ventajas de estos tres modelos es que son de uso libre, y que todos aproximan una solución a las ecuaciones de hidrodinámica promediadas en la profundidad sobre mallas estructuradas y no estructuradas formadas por elementos de tres (triángulos), cuatro (cuadriláteros) o más lados, e incluyen algunos modelos de turbulencia. 
El trabajo se integra de cinco apartados. En el primero se describe el conjunto experimental y el funcionamiento hidráulico del mismo, el equipo de medición empleado y las pruebas realizadas. En el segundo se hace una breve descripción de los sistemas de modelación utilizados, así como de algunas de sus características principales. En el tercero se presentan los resultados y una comparación gráfica entre los componentes de la velocidad medidos $\left(\begin{array}{lllll}\bar{u} & \bar{v}\end{array}\right)$ y los calculados numéricamente (la barra indica promedios en el tiempo). En el cuarto se realiza un análisis de resultados y se presentan las diferencias de la comparación. Finalmente, en el quinto se incluyen las conclusiones y recomendaciones para futuros trabajos.

\section{Materiales y métodos}

\section{Conjunto experimental}


Tecnología y

Ciencias $₫$ Agua
2022, Instituto Mexicano de Tecnología del Agua

Open Access bajo la licencia CC BY-NC-SA 4.0

(https://creativecommons.org/licenses/by-nc-sa/4.0/)

El dispositivo experimental es un canal de sección rectangular con curvatura compuesta (curva mixta cóncava-convexa-cóncava con radios de curvatura de $1.67,0.725$ y 0.964 m, respectivamente), pendiente horizontal, ancho variable y una longitud horizontal de $6.53 \mathrm{~m}$ (Figura 1).

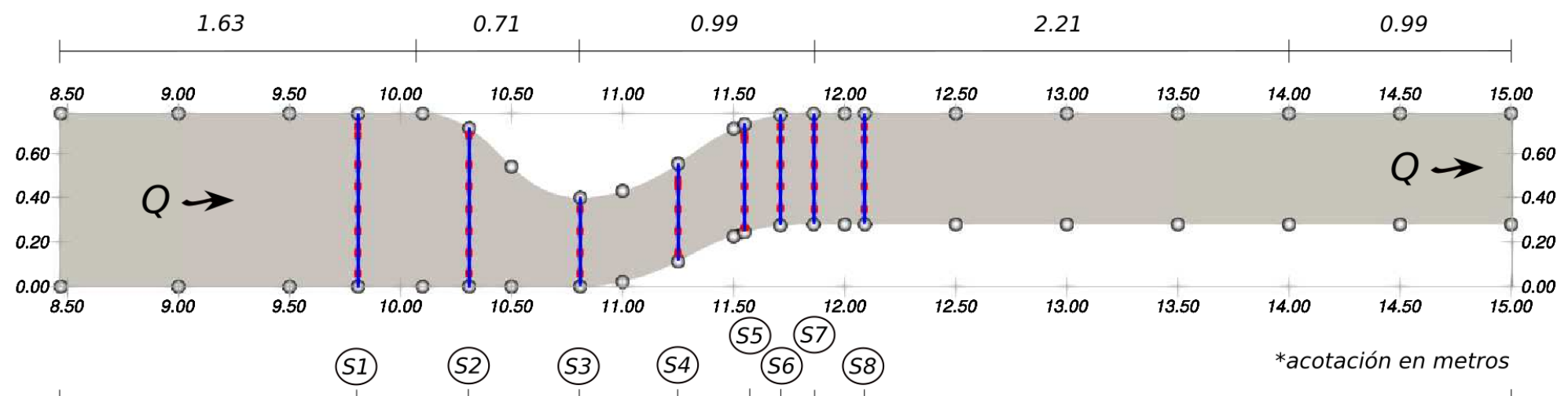

Figura 1. Geometría y dimensiones del canal en planta.

El canal inicia con un tramo recto de $1.63 \mathrm{~m}$ de longitud y ancho de plantilla constante $(b=0.78 \mathrm{~m})$. En el trazo en planta, en la margen izquierda (MI) empieza la reducción del ancho por la presencia de la curva cóncava, la cual llega a un ancho mínimo $(b=0.40 \mathrm{~m})$; hasta este mismo punto, la margen derecha (MD) se mantiene recta. Después se presenta una curva convexa sobre ambas márgenes; enseguida el canal retoma una curvatura cóncava hasta alcanzar aguas abajo la trayectoria recta inicial con ambas márgenes paralelas. 
Teçnología y

Ciencias $₫$ Agua
2022, Instituto Mexicano de Tecnología del Agua

Open Access bajo la licencia CC BY-NC-SA 4.0

(https://creativecommons.org/licenses/by-nc-sa/4.0/)

El ancho de la sección posterior a la máxima contracción $(b=0.40 \mathrm{~m})$ presenta una ampliación gradual de 0.40 a $0.50 \mathrm{~m}$ sobre la MI. La longitud recta de la salida del canal es de $3.20 \mathrm{~m}$. La plantilla del canal es de acero y las paredes laterales son de vidrio. El tramo del canal que se estudia comprende desde $X=9.50 \mathrm{~m}$ hasta $X=13.0 \mathrm{~m}$, con un área en planta de $1.932 \mathrm{~m}^{2}$ y un perímetro de $8.539 \mathrm{~m}$. Se fijaron ocho secciones transversales en las que se realizan las mediciones puntuales de la velocidad, localizadas perpendicularmente al eje $X$ (Figura 1 y Tabla 1 ).

Tabla 1. Secciones de medición, localización y ancho.

\begin{tabular}{|c|c|c|}
\hline $\begin{array}{c}\text { Sección } \\
{[I D]}\end{array}$ & $\begin{array}{c}\boldsymbol{A} \\
{[\mathrm{m}]}\end{array}$ & $\begin{array}{c}\text { Ancho } \\
{[\mathrm{m}]}\end{array}$ \\
\hline S1 & 9.81 & 0.780 \\
\hline S2 & 10.31 & 0.713 \\
\hline S3 & 10.81 & 0.400 \\
\hline S4 & 11.25 & 0.441 \\
\hline S5 & 11.55 & 0.467 \\
\hline S6 & 11.71 & 0.478 \\
\hline S7 & 11.86 & 0.499 \\
\hline
\end{tabular}




\begin{tabular}{|l|l|l|}
\hline S8 & 12.09 & 0.500 \\
\hline
\end{tabular}

Cuando el ancho del canal es menor a $0.749 \mathrm{~m}$, éste se podría considerar estrecho $(B / h<5)$ y con efectos de pared, pero debido al material de las paredes (vidrio) y fondo (acero), se trata de un canal de pared hidráulicamente liso y con rugosidades similares, por lo que no es necesario realizar el análisis de dicho efecto en la resistencia al flujo producido por la fricción de las paredes en el campo de velocidades, pues se considera despreciable (Guo, 2017).

Las mediciones puntuales de los tres componentes de la velocidad se obtuvieron con un velocímetro Doppler acústico (Micro-ADV $16 \mathrm{MHz}$ ), orientado en la dirección vertical (con los sensores hacia abajo). La descripción detallada de la operación del equipo de medición se encuentra en Kraus, Lohrmann y Cabrera (1994); Lohrmann, Cabrera y Kraus (1994); Anderson y Lohrmann (1995); Lane et al. (1998); Voulgaris y Trowbridge (1998); Nikora y Goring (1998); McLelland y Nicholas (2000), y López y Garcia (2001), entre otros. El ADV se colocó en un soporte de acero que permite desplazar el instrumento tanto en el sentido horizontal como en el vertical, con la finalidad de controlar la posición de los sensores dentro del flujo y determinar su posición relativa con respecto a una margen del canal. Los datos recolectados durante un periodo de tiempo y con cierta frecuencia se analizaron con el software WinADV (Wahl, 2000), primero para determinar los parámetros de calidad y posteriormente las características del flujo. 
La calidad de los datos recopilados se define con dos parámetros: la relación señal-ruido (SNR, por sus siglas en inglés) y el coeficiente de correlación (COR) para cada receptor del ADV; el fabricante recomienda valores de SNR superiores a $15 \mathrm{~dB}$ y coeficiente de correlación mayor al $70 \%$ para tener una descripción adecuada de las características del flujo y de la turbulencia; estos parámetros dependen de partículas y/o microburbujas que viajan con el fluido o de la adición de partículas de siembra (SonTek, 1997). En los experimentos, los datos se filtraron con SNR superior a $20 \mathrm{~dB}$ y $90 \%$ de correlación, por lo que en este estudio en particular no fue necesario introducir ningún tipo de partículas de siembra para mejorar las mediciones debido a la naturaleza turbulenta del flujo.

\section{Prueba experimental}

La campaña de medición de la prueba A (Tabla 2) consiste en establecer un caudal de entrada de $Q=10.3 \mathrm{l} / \mathrm{s}$ suministrado por un depósito de carga constante, abastecido por dos equipos de bombeo de 5.0 hp cada uno y aforado con un vertedor triangular de pared delgada con un ángulo de $90^{\circ}$ situado en $X=0.00 \mathrm{~m}$ (el caudal de salida llega a un cárcamo de 
Teçnología y

Ciencias $₫$ Agua
2022, Instituto Mexicano de Tecnología del Agua

Open Access bajo la licencia CC BY-NC-SA 4.0

(https://creativecommons.org/licenses/by-nc-sa/4.0/)

bombeo subterráneo generando un circuito cerrado); y un tirante de $y=$ $0.149 \mathrm{~m}$ localizado en $X=13.00 \mathrm{~m}$ establecido mediante una compuerta vertical plana ubicada en $X=15 \mathrm{~m}$; las condiciones anteriores permitieron establecer en el canal un flujo subcrítico y permanente.

Tabla 2. Condición experimental.

\begin{tabular}{|c|c|c|c|c|c|c|c|}
\hline Prueba & $\begin{array}{c}\boldsymbol{Q} \\
{[\boldsymbol{l} / \boldsymbol{s}]}\end{array}$ & $\begin{array}{c}\boldsymbol{y} \\
{[\boldsymbol{m}]}\end{array}$ & $\begin{array}{c}\boldsymbol{U}_{\boldsymbol{s 1}} \\
{[\boldsymbol{m} / \boldsymbol{s}]}\end{array}$ & $\begin{array}{c}\boldsymbol{S}_{\boldsymbol{w}} \\
{\left[\boldsymbol{x \mathbf { 1 0 } ^ { - 4 } ]}\right.}\end{array}$ & $\begin{array}{c}\boldsymbol{F}_{\boldsymbol{s}_{\boldsymbol{s}}} \\
-\end{array}$ & $\begin{array}{c}\boldsymbol{R}_{\boldsymbol{e}_{\boldsymbol{s} 1}} \\
{\left[\boldsymbol{x \mathbf { 1 0 } ^ { 4 } ]}\right.}\end{array}$ & $\begin{array}{c}\boldsymbol{h}_{\boldsymbol{m}} \\
{[\mathrm{m}]}\end{array}$ \\
\hline $\mathrm{A}$ & 10.3 & 0.149 & 0.088 & 4.3 & 0.072 & 1.32 & 0.06 \\
\hline
\end{tabular}

El volumen de muestreo para determinar los componentes de la velocidad se ubicó a una elevación del $40 \%$ del tirante, es decir, $h_{m}=$ $0.4 y$, medido desde la plantilla del canal, esto es, a $0.06 \mathrm{~m}$ (Tabla 2). La medición de los componentes de la velocidad se realizó en 45 puntos (Tabla 3), con una frecuencia de muestreo de $50 \mathrm{~Hz}$ durante $180 \mathrm{~s}$. Con la información obtenida de los ocho puntos de medición de la sección S1 se determinaron las variables hidráulicas indicadas en la Tabla 2.

Tabla 3. Puntos de medición.

\begin{tabular}{|l|l|l|l|l|l|l|l|}
\hline Punto & Sección & $X$ & $Y$ & Punto & Sección & $X$ & $Y$ \\
\hline
\end{tabular}


Tecnología y

Ciencias 홈ua
2022, Instituto Mexicano de Tecnología del Agua

Open Access bajo la licencia CCBY-NC-SA 4.0

(https://creativecommons.org/licenses/by-nc-sa/4.0/)

\begin{tabular}{|c|c|c|c|c|c|c|c|}
\hline & & {$[m]$} & {$[m]$} & & & {$[\mathrm{m}]$} & {$[m]$} \\
\hline 1 & $\mathrm{~S} 1$ & 9.81 & 0.720 & 24 & S4 & 11.25 & 0.038 \\
\hline 2 & $\mathrm{~S} 1$ & 9.81 & 0.680 & 25 & S5 & 11.55 & 0.436 \\
\hline 3 & $\mathrm{~S} 1$ & 9.81 & 0.550 & 26 & S5 & 11.55 & 0.416 \\
\hline 4 & $\mathrm{~S} 1$ & 9.81 & 0.450 & 27 & S5 & 11.55 & 0.306 \\
\hline 5 & $\mathrm{~S} 1$ & 9.81 & 0.350 & 28 & S5 & 11.55 & 0.206 \\
\hline 6 & $\mathrm{~S} 1$ & 9.81 & 0.250 & 29 & S5 & 11.55 & 0.106 \\
\hline 7 & $\mathrm{~S} 1$ & 9.81 & 0.150 & 30 & S5 & 11.55 & 0.021 \\
\hline 8 & $\mathrm{~S} 1$ & 9.81 & 0.060 & 31 & S6 & 11.71 & 0.446 \\
\hline 9 & $\mathrm{~S} 2$ & 10.31 & 0.680 & 32 & S6 & 11.71 & 0.386 \\
\hline 10 & S2 & 10.31 & 0.550 & 33 & S6 & 11.71 & 0.276 \\
\hline 11 & $\mathrm{~S} 2$ & 10.31 & 0.450 & 34 & S6 & 11.71 & 0.176 \\
\hline 12 & $\mathrm{~S} 2$ & 10.31 & 0.350 & 35 & S6 & 11.71 & 0.076 \\
\hline 13 & $\mathrm{~S} 2$ & 10.31 & 0.250 & 36 & S7 & 11.86 & 0.440 \\
\hline 14 & S2 & 10.31 & 0.150 & 37 & S7 & 11.86 & 0.380 \\
\hline
\end{tabular}


Tecnología y

Ciencias $\approx$ Agua
2022, Instituto Mexicano de Tecnología del Agua

Open Access bajo la licencia CC BY-NC-SA 4.0

(https://creativecommons.org/licenses/by-nc-sa/4.0/)

\begin{tabular}{|c|c|c|c|c|c|c|c|}
\hline 15 & S2 & 10.31 & 0.060 & 38 & S7 & 11.86 & 0.270 \\
\hline 16 & S3 & 10.81 & 0.350 & 39 & S7 & 11.86 & 0.170 \\
\hline 17 & S3 & 10.81 & 0.250 & 40 & S7 & 11.86 & 0.070 \\
\hline 18 & S3 & 10.81 & 0.150 & 41 & S8 & 12.09 & 0.440 \\
\hline 19 & S3 & 10.81 & 0.060 & 42 & S8 & 12.09 & 0.370 \\
\hline 20 & S4 & 11.25 & 0.368 & 43 & S8 & 12.09 & 0.280 \\
\hline 21 & S4 & 11.25 & 0.338 & 44 & S8 & 12.09 & 0.170 \\
\hline 22 & S4 & 11.25 & 0.238 & 45 & S8 & 12.09 & 0.070 \\
\hline 23 & S4 & 11.25 & 0.138 & & & & \\
\hline
\end{tabular}

En la Tabla 2, $Q$ es el caudal de entrada; $y$, el tirante localizado en $X=13.00 \mathrm{~m} ; U_{S 1}$, la velocidad media medida en la sección S1 (velocidad de referencia para los cálculos presentados más adelante), la cual se obtuvo como el promedio aritmético de la magnitud de la velocidad de cada uno de los ocho puntos de medición de la sección $\mathrm{S} 1 ; S_{w}$, la pendiente de la superficie libre del agua entre las secciones S1-S8; $h_{m}$, la elevación de medición a partir del fondo; $F_{r_{s 1}}$ y $R_{e_{s 1}}$ son el número de Froude y Reynolds en la sección S1, respectivamente. 
A partir de las mediciones de velocidad y el tirante de agua se determina que la superficie libre del agua tiende a ser horizontal, como se indica en la Tabla 2. También se observó que en la margen izquierda del canal, cerca de la sección S4, se forma una estela de pequeños remolinos apreciable en la superficie libre del agua.

\section{Modelos matemáticos}

Se presenta una breve descripción de los modelos matemáticos utilizados para el cálculo de la hidrodinámica 2DH, así como las referencias en las que se puede profundizar más en el tema.

\section{HEC-RAS}


El sistema de modelación matemática HEC-RAS (Hydrologic Engineering Center-River Analysis System) es desarrollado por el Centro de Ingenieńa Hidrológica (Hydrologic Engineering Center) del Instituto de Recursos Hídricos (Institute Water Resources) del Cuerpo de Ingenieros de los Estados Unidos (US Army Corps of Engineers). Este sistema permite realizar el cálculo de la hidrodinámica en flujos a superficie libre con régimen permanente y variable, en una y dos dimensiones, transporte de sedimentos y análisis de calidad del agua en redes de canales y ríos (HECRAS, 2016c).

El módulo de cálculo de flujo variable en dos dimensiones resuelve las ecuaciones de la onda difusiva y/o las ecuaciones de Saint Venant, con la opción de utilizar el modelo de turbulencia parabólico. Las ecuaciones son aproximadas con los métodos de diferencias finitas y volúmenes finitos de tipo implícito, que permite pasos de tiempo de cálculo mayores que los de tipo explícito, y un algoritmo de secado-mojado robusto; las mallas de cálculo pueden ser estructuradas y no estructuradas hasta con ocho lados (HEC-RAS, 2016a; HEC-RAS, 2016b). En este trabajo se utilizó el módulo correspondiente al cálculo de flujo bidimensional horizontal con las ecuaciones de Saint Venant y el modelo de turbulencia parabólico.

Mediante RAS Mapper se define la zona de estudio y se elabora la malla de cálculo (tipo, tamaño y número de elementos); posteriormente se imponen las condiciones hidráulicas, iniciales y de contorno, finalmente se definen los datos de cálculo. 


\section{Iber}

Iber es un modelo matemático bidimensional para la simulación del flujo a superficie libre, morfodinámica, procesos de transporte y hábitat en ríos y estuarios, desarrollado a partir de la colaboración del Grupo de Ingeniería del Agua y del Medio Ambiente, GEAMA (Universidade da Coruña), del Grupo de Ingeniería Matemática (Universidade de Santiago de Compostela), del Instituto Flumen (Universitat Politècnica de Catalunya y Centre Internacional de Mètodes Numèrics a I'Enginyeria) y promovido por el Centro de Estudios Hidrográficos del CEDEX (Bladé et al., 2014b). Consta de diferentes módulos acoplados, entre ellos, los módulos de hidrodinámica y de turbulencia, los cuales se emplean en este trabajo.

El módulo de hidrodinámica resuelve las ecuaciones bidimensionales de aguas poco profundas promediadas en la profundidad (SWE) para calcular la profundidad del agua y los dos componentes horizontales de la velocidad (Bladé et al., 2014b); en el módulo de turbulencia se incluyen varios modelos, uno es el de tipo Boussinesq, otro es el promediado en la profundidad para calcular los esfuerzos de corte turbulentos (Cea, Puertas, \& Vázquez-Cendón, 2007), y también el modelo $k-\varepsilon$ (Rastogi \& Rodi, 1978). Estas ecuaciones se resuelven con 
un método de volúmenes finitos de tipo explícito en el tiempo sobre mallas estructuradas y/o no estructuradas con elementos de 3 y/o 4 lados (Bladé et al., 2014b). El preproceso de los datos y posproceso de los resultados se realiza mediante la herramienta GiD (CIMNE, 2009).

En el preproceso de datos, mediante la interfaz de GiD se elabora la geometría de la zona de estudio, y se imponen las condiciones hidráulicas, condiciones iniciales y de contorno; posteriormente se define el tipo, tamaño y número de elementos (malla de cálculo), y por último se determinan los datos de cálculo.

\section{Telemac-Mascaret}

El sistema Telemac-Mascaret en su versión 2DH permite aproximar una solución a las ecuaciones de flujo a superficie libre promediadas en la profundidad (Hervouet, 2007). Se basa esencialmente en la técnica de elementos finitos y permite determinar magnitudes hidráulicas que son dependientes de la geometría. El dominio de cálculo se discretiza con elementos triangulares, y el cálculo lo desarrolla en cada nodo de la malla; también permite incorporar los efectos de la turbulencia mediante el modelo $k-\varepsilon$ (Rastogi \& Rodi, 1978); cuenta con módulos adicionales 
como lo son calidad del agua y transporte de sedimentos, entre otros (EDF-R\&D, 2016).

En la etapa de preproceso se construye la geometría del modelo (canal curvo) y se establecen las condiciones de frontera; para esto se emplea la herramienta Blue Kenue ( $\mathrm{CHC}$, 2011), con la que se realiza el preproceso y posproceso. Las ecuaciones de flujo y de turbulencia se resuelven de forma modular con el método de pasos fraccionados: primero se calcula la advección con el método de las características, luego la propagación y la difusión (incluida la turbulencia) y los términos fuente en las ecuaciones dinámicas, aunque existen recomendaciones para implementar los esquemas numéricos (Hervouet, 2007).

En los tres modelos, las ecuaciones 2DH consideran la hipótesis de distribución de presión hidrostática y velocidad uniforme en la profundidad del agua, ; estas hipótesis son bien aceptadas en la aplicación en la ingeniería de ríos.

\section{Resultados}


La discretización del canal curvo con HEC-RAS se realizó con 23559 elementos de 4 y 5 lados con longitud, y área promedio de $0.009 \mathrm{~m} \mathrm{y}$ $0.818 \mathrm{~cm}^{2}$, respectivamente; en Iber con 1277640 elementos de cuatro lados con longitud de 0.001-0.0015 m y área promedio de $0.015 \mathrm{~cm}^{2}$, respectivamente; y con Telemac-Mascaret con 153149 elementos de tres lados con longitud de 0.001-0.005 m y área promedio de $0.0126 \mathrm{~cm}^{2}$, respectivamente. El tipo de malla utilizado en cada modelo se indica en la Tabla 4.

Tabla 4. Datos de cálculo.

\begin{tabular}{|c|c|c|c|c|c|}
\hline Modelo & $\begin{array}{c}\text { Tipo de } \\
\text { malla }\end{array}$ & $\begin{array}{c}\text { Núm. de } \\
\text { elementos }\end{array}$ & $\begin{array}{c}\text { Modelo de } \\
\text { turbulencia }\end{array}$ & CFL & $\begin{array}{c}\Delta \mathbf{t} \\
(\mathbf{s})\end{array}$ \\
\hline HEC-RAS & Estructurada & 23559 & Parabólico & - & 0.10 \\
\hline Iber & Estructurada & 1277640 & $\mathrm{k}-\varepsilon$ & 0.45 & $\begin{array}{c}0.00016- \\
0.0019\end{array}$ \\
\hline Telemac- & $\begin{array}{c}\text { No } \\
\text { Mascaret }\end{array}$ & 153149 & $\mathrm{k}-\varepsilon$ & 0.10 & 0.0015 \\
\hline
\end{tabular}

En los tres modelos se usó como condición inicial una profundidad de $0.149 \mathrm{~m}$; en la condición de contorno de entrada un caudal de 0.0105 $\mathrm{m}^{3} / \mathrm{s}$ y de salida un tirante de $0.149 \mathrm{~m}$. La fricción del fondo y las paredes 
se consideró mediante un único coeficiente de fricción de Manning de $0.011 \mathrm{~s} / \mathrm{m}^{1 / 3}$; dicho parámetro se empleó para calibrar los modelos, esto es, se propusieron distintos valores del coeficiente de fricción de Manning hasta obtener las diferencias mínimas entre los valores de velocidad calculada y medida. Con respecto al incremento de tiempo de cálculo en HEC-RAS se utilizó el mínimo permitido de 0.10 s, en Iber y TelemaMascaret, los determinados por la condición de Courant-Friedrichs-Levy (CFL), que corresponden a 0.0016 s-0.00019 s y 0.0015 s, respectivamente. El modelo de turbulencia utilizado en cada modelo se muestra en la Tabla 4.

Con las condiciones anteriores se realizó el cálculo hasta alcanzar las condiciones de flujo permanente, lo cual se logró con un tiempo de seis minutos; de esta forma, los tiempos de cálculo fueron de 10 minutos con HEC-RAS, en un equipo de cómputo con un procesador Intel Core i74770, 3.4 GHz, $16 \mathrm{~GB}$ de RAM; de 35.38 horas con Iber en un tarjeta gráfica GeForce GTX 650 Ti de un equipo de cómputo con un procesador Intel Core i7-4770, 3.4 GHz, 16 GB de RAM; en el sistema Telemac, la optimización del proceso de cálculo distribuido se desarrolló en un clúster con 24 núcleos de procesamiento AMD, distribución CentOS, versión 6.9, arquitectura de 64 bits; para el cálculo en paralelo se utilizó la interfaz de envió de mensajes (MPI), que permite el intercambio de mensajes entre Ios procesadores (Gropp, Lusk, \& Skijellum, 2014) y METIS, que realiza la distribución de la malla entre el número de procesadores, permitiendo que el número de elementos de la malla sea el mismo para cada procesador y así minimizar el número de elementos adyacentes, esto con 
el objetivo de equilibrarel cálculo entre los procesadores (Karypis, 2013), por lo que el tiempo de cálculo fue de 3.67 horas.

En la Figura 2 se presenta la comparación de los resultados calculados con los datos medidos. En cada ilustración, el eje de las abscisas corresponde a los componentes de la velocidad $\bar{u}$ (izquierda) y $\bar{v}$ (derecha) adimensionalizadas con la velocidad media de la sección S1 (velocidad de referencia); el eje de las ordenadas corresponde a la ubicación en planta del punto de medición adimensionalizada con el ancho total de la sección transversal del canal. 
Tecnología y

Ciencias $\approx$ Agua
2022, Instituto Mexicano de Tecnología del Agua

Open Access bajo la licencia CC BY-NC-SA 4.0

(https://creativecommons.org/licenses/by-nc-sa/4.0/)
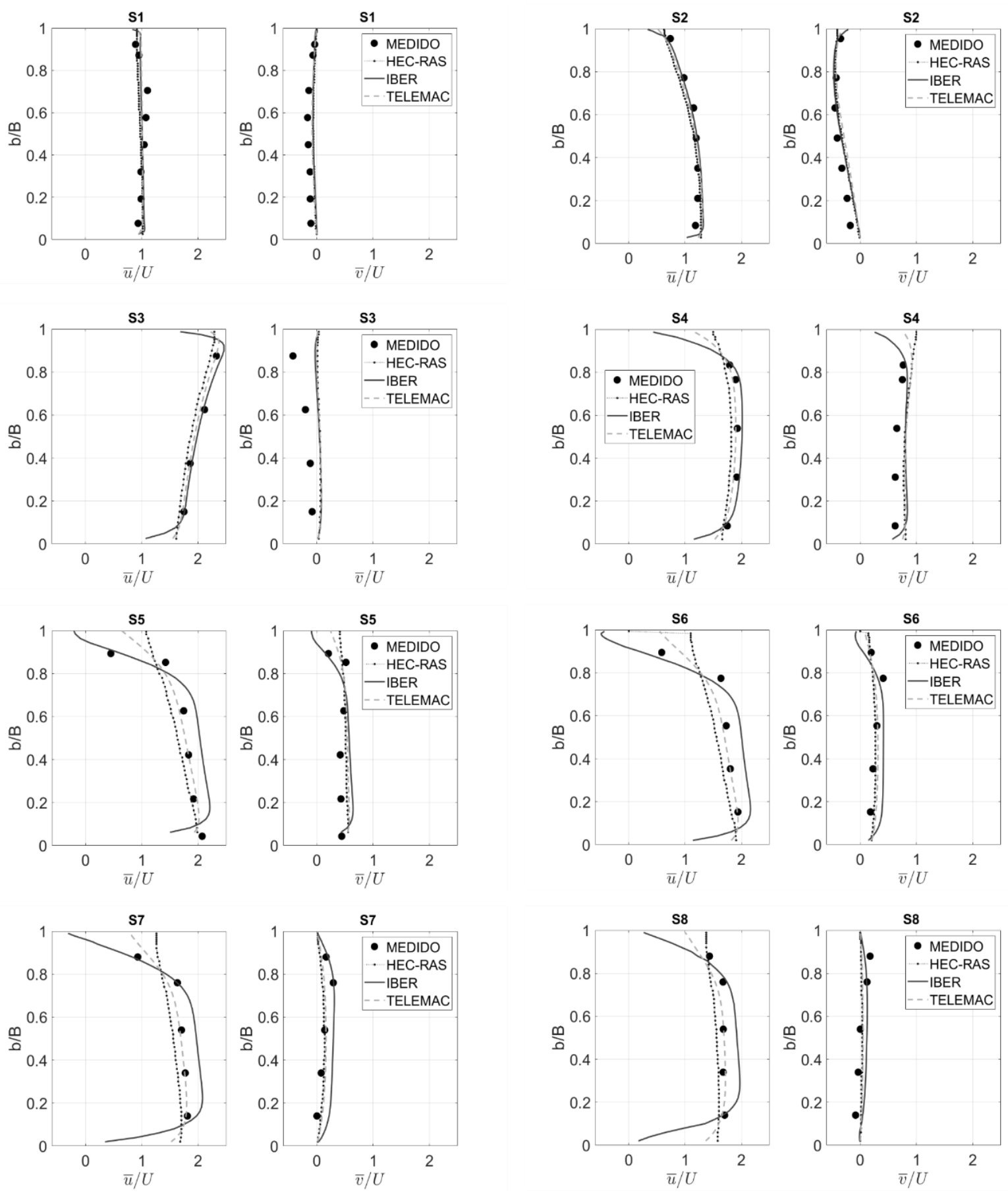

Figura 2. Comparación numérico-experimental de los componentes horizontales de la velocidad. 
Tecnología y

Ciencias $₫$ Agua
2022, Instituto Mexicano de Tecnología del Agua

Open Access bajo la licencia CCBY-NC-SA 4.0

(https://creativecommons.org/licenses/by-nc-sa/4.0/)

Además, para profundizar en la comprensión de la hidrodinámica del fenómeno que se presenta entre las secciones S3 y S7, se presentan los campos de los componentes de la velocidad adimensionalizados con la velocidad media de la sección $\mathrm{S} 1$ (velocidad de referencia) $\bar{u} / U_{S 1}$ (Figura 3a) y $\bar{v} / U_{S 1}$ (Figura $3 \mathrm{~b}$ ); la magnitud y gradiente de la velocidad (Figura 4), que permite mostrar el desarrollo de la capa de corte; la energía cinética de la turbulencia (Figura 5) y el número de Reynolds (Figura 6); todos ellos calculados por el modelo Telemac-Mascaret.

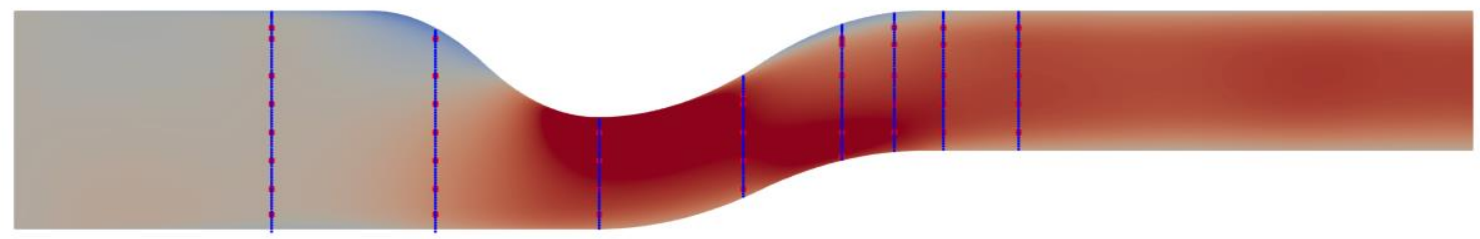

$\Delta Y$

$7 x$

$\begin{array}{lllllllllllllllllllll}0.0 & 0.1 & 0.2 & 0.3 & 0.4 & 0.5 & 0.6 & 0.7 & 0.8 & 0.9 & 1.0 & 1.1 & 1.2 & 1.3 & 1.4 & 1.5 & 1.6 & 1.7 & 1.8 & 1.9 & 2.0\end{array}$

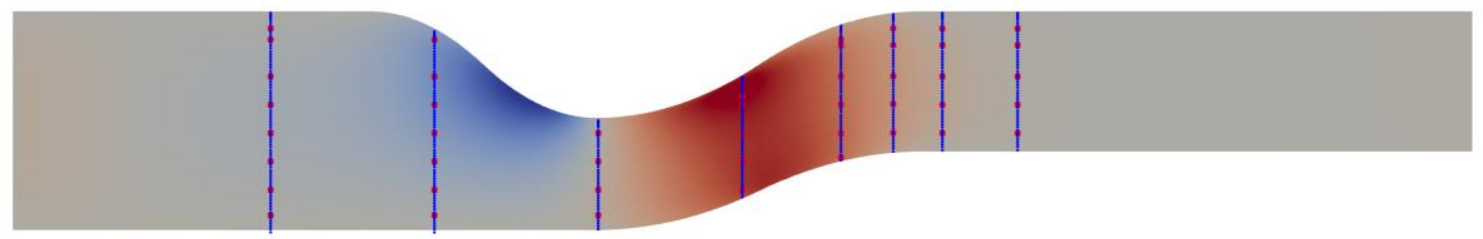

$\Delta Y$

$2 x$

$\mathrm{V} / \mathrm{U}$

$\begin{array}{lllllllllllllllllllll}-1.0 & -0.9 & -0.8 & -0.7 & -0.6 & -0.5 & -0.4 & -0.3 & -0.2 & -0.1 & 0.0 & 0.1 & 0.2 & 0.3 & 0.4 & 0.5 & 0.6 & 0.7 & 0.8 & 0.9 & 1.0\end{array}$

Figura 3. Campos de los componentes de la velocidad. 
Tecnología y
2022, Instituto Mexicano de Tecnología del Agua

Open Access bajo la licencia CC BY-NC-SA 4.0

(https://creativecommons.org/licenses/by-nc-sa/4.0/)

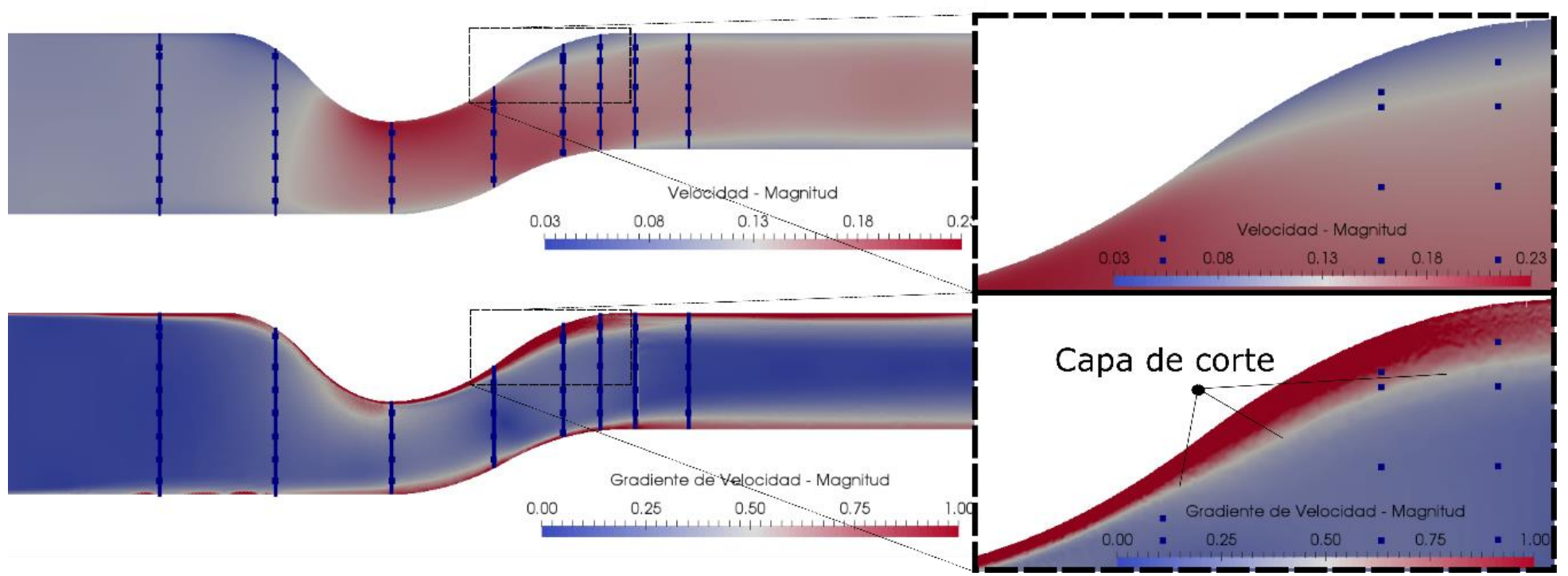

Figura 4. Magnitud y gradiente de la velocidad.

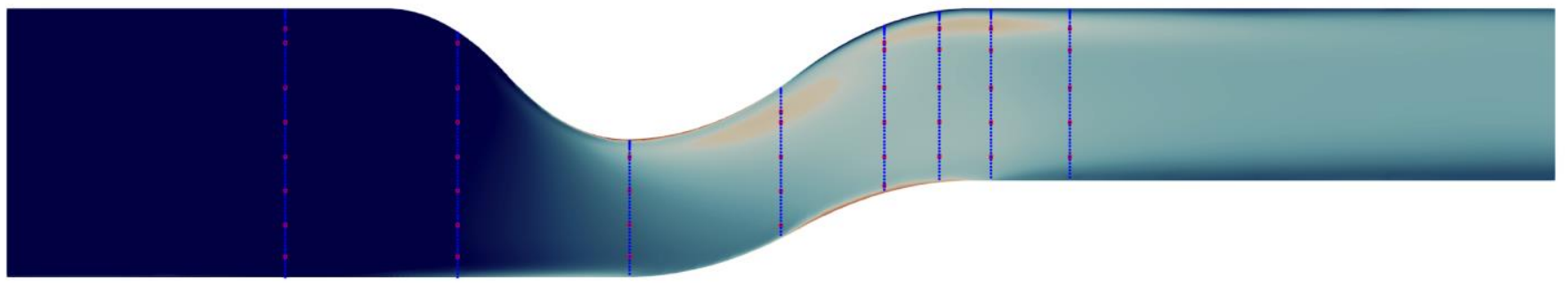

Figura 5. Energía cinética de la turbulencia. 
Tecnología y

Ciencias $₫$ Agua
2022, Instituto Mexicano de Tecnología del Agua

Open Access bajo la licencia CCBY-NC-SA 4.0

(https://creativecommons.org/licenses/by-nc-sa/4.0/)

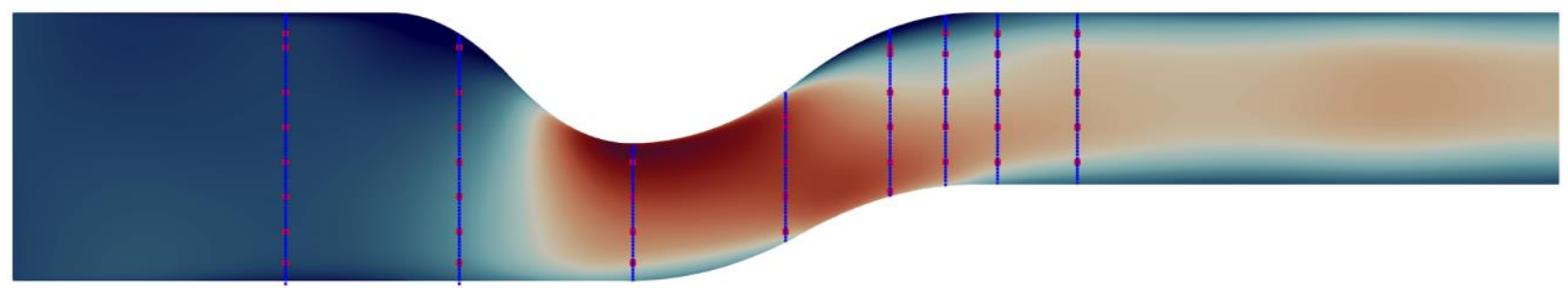

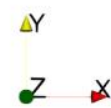

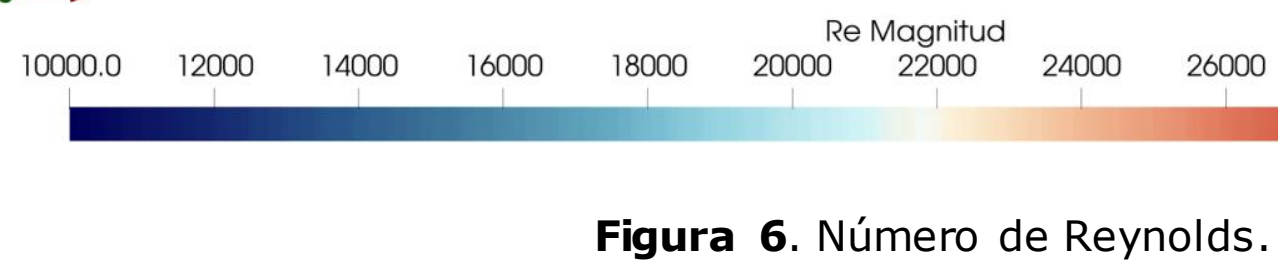

\section{Análisis y discusión de resultados}

De forma general, los resultados calculados con los modelos empleados siguen la tendencia de los valores medidos. Así, en las primeras dos secciones (S1 y S2), los valores medidos y calculados son similares; en cambio, en las siguientes dos secciones (S3 y S4), dicha similitud se 
mantiene solamente en el componente $\bar{u}$ de la velocidad, dado que en el componente $\bar{v}$, los tres modelos tienen un comportamiento distinto a los valores medidos. En las últimas cuatro secciones transversales, a pesar de que los valores calculados siguen la tendencia de los valores medidos, existe mayor dispersión de éstos, pero los resultados fueron ligeramente mejores que los reportados por Jiménez y Berezowsky (2004). En todas las secciones transversales se observa que los resultados del modelo Telemac-Mascaret siempre se encuentran entre los resultados de HECRAS e Iber; con HEC-RAS se obtienen valores menores a los medidos en la margen derecha y mayores en la margen izquierda; en cambio, con Iber, el comportamiento de los valores es a la inversa, presumiblemente debido a que trata de adaptarse al perfil de velocidades cerca de las paredes; en dichos resultados no se percibe una mejora debido al aumento del número de elementos, lo cual es compatible con los resultados reportados por Ghamry y Steffler (2005) en canales curvos.

Con la finalidad de conocer el desempeño de cada uno de los modelos empleados se realizó un análisis de errores entre los valores medidos y calculados. Determinar la diferencia de la comparación numérico-experimental es una tarea compleja, ya que siempre existen errores innatos en el proceso de medición y los asociados con la calibración de los modelos, la instrumentación y/o los equipos de medición. Aunado a lo anterior, se encuentran los procesos hidráulicos y turbulentos que intervienen en el comportamiento del flujo, recordemos que actualmente la turbulencia sigue siendo un fenómeno sin resolver. 
Tecnología y

Ciencias $₫$ Agua
2022, Instituto Mexicano de Tecnología del Agua

Open Access bajo la licencia CCBY-NC-SA 4.0

(https://creativecommons.org/licenses/by-nc-sa/4.0/)

Por otra parte, la medición de una variable disminuye la incertidumbre sobre su comportamiento siempre y cuando se sigan ciertos criterios, con el objetivo de minimizar los distintos tipos de errores; al tener un registro en el tiempo de su comportamiento y al procesar dicha información, se puede tener en cuenta qué parte del registro de medición es aceptable y cuál no mediante parámetros de calidad (SNR y COR).

De esta forma, con la finalidad de determinar la aproximación que ofrece cada uno de los modelos numéricos, se plantea un análisis de regresión lineal simple con el fin de estimar la relación entre la velocidad medida y la calculada por los modelos numéricos utilizados. Partiendo desde el punto de vista de que en las mediciones se pueden presentar distintos tipos de errores innatos en el proceso de medición y que los modelos numéricos son aproximaciones a la solución de ecuaciones, en este caso en particular 2DH o SWE, y que no son una solución exacta del comportamiento de un flujo a superficie libre, y adicionalmente a la hipótesis de que la variación del comportamiento de la distribución de velocidades en la vertical puede ser reemplazada por un promedio, se puede establecer la relación indicada.

En la Figura 7 se presenta dicho análisis para los 45 puntos de medición de las tres variables de la velocidad $(\bar{U}, \bar{u}$ y $\bar{v})$; los resultados se presentan adimensionalizados como: $\quad \bar{U}_{\text {med }}=\frac{|\bar{U}|_{\text {medida }}}{U_{S 1}}, \quad \bar{U}_{c a l}=\frac{\mid \overline{|U|_{\text {calculada }}}}{U_{S 1}}$, $\bar{u}_{x_{\text {med }}}=\frac{\bar{u}_{x_{\text {medida }}}}{U_{S 1}}, \bar{u}_{y_{\text {med }}}=\frac{\bar{u}_{y_{\text {medida }}}}{U_{S 1}}, \bar{u}_{x_{\text {cal }}}=\frac{\bar{u}_{x_{\text {calculada }}}}{U_{S 1}}, \bar{u}_{y_{\text {cal }}}=\frac{\bar{u}_{y_{\text {calculada }}} \text {. Con línea }}{U_{S 1}}$ continua se representa la relación esperada (RE) considerada como óptima, la cual indica que la velocidad medida debe ser igual a la velocidad 
calculada o viceversa; los puntos corresponden a la relación entre la velocidad medida y la calculada (RMC); finalmente, se determina y se muestra la correlación existente mediante regresión lineal simple con línea discontinua (RL) y su correspondiente coeficiente de correlación (R), que permite determinar la bondad de ajuste del modelo de regresión (Menhenhall, Beaver, \& Beaver, 2010). En la Tabla 5 se presentan los coeficientes de correlación R. 
Tecnología y

Ciencias $₫$ Agua
2022, Instituto Mexicano de Tecnología del Agua

Open Access bajo la licencia CC BY-NC-SA 4.0

(https://creativecommons.org/licenses/by-nc-sa/4.0/)
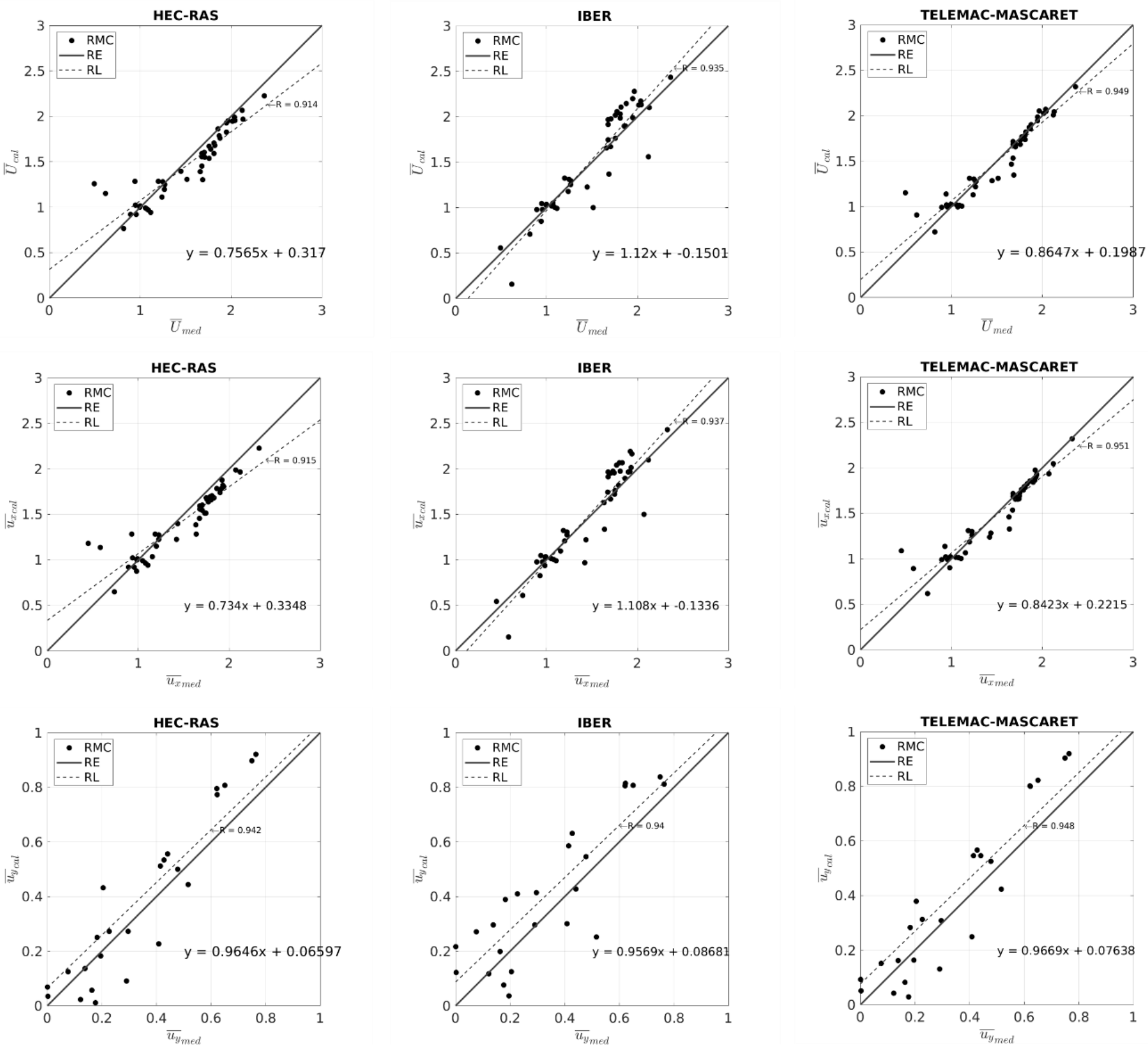

Figura 7. Modelos de regresión lineal. 
Tabla 5. Coeficientes de correlación para los 45 puntos de medición.

\begin{tabular}{|c|c|c|c|}
\hline Modelo/variable & $\boldsymbol{R}_{\bar{U}}$ & $\boldsymbol{R}_{\overline{\mathbf{u}}}$ & $\boldsymbol{R}_{\bar{v}}$ \\
\hline HEC-RAS & 0.914 & 0.915 & 0.942 \\
\hline Iber & 0.935 & 0.937 & 0.940 \\
\hline Telemac-Mascaret & 0.949 & 0.951 & 0.948 \\
\hline
\end{tabular}

A partir del análisis anterior, se observa que los menores valores de correlación corresponden a HEC-RAS, mientras que los mayores a Telemac-Mascaret; en cambio, Iber está muy cerca del valor medio, aunque para fines ingenieriles los tres modelos son aceptables.

El procedimiento descrito anteriormente también se realizó para los puntos de medición de las tres variables de la velocidad de cada sección; los coeficientes de correlación se presentan en la Tabla 6. De forma particular se destaca que con los tres modelos en la sección S1 se presentan las menores correlaciones, siendo muy bajas para la magnitud de la velocidad y los componentes; al revisar los ajustes en esta sección se observa que los modelos tienden a subestimar para una velocidad mayor a la unidad y sobreestimar para una velocidad menor a la unidad, esto tiene que ver con el proceso de remover la velocidad media, ya que con ello quedan pequeñas fluctuaciones que no tienden a ajustarse a un modelo lineal con una recta a $45^{\circ}$ sino más bien a una recta horizontal con una ordenada al origen muy cercana a la unidad, además es la sección 
Tecnología y

Ciencias Agua
2022, Instituto Mexicano de Tecnología del Agua

Open Access bajo la licencia CC BY-NC-SA 4.0

(https://creativecommons.org/licenses/by-nc-sa/4.0/)

en la cual se determina la velocidad de referencia; la siguiente sección con menor coeficiente de correlación con los tres modelos corresponde a la sección S5. También se observa que con HEC-RAS se obtienen coeficientes de correlación bajos en el componente de velocidad $\bar{v}$.

Tabla 6. Coeficientes de correlación para los puntos de cada sección de medición.

\begin{tabular}{|c|c|c|c|c|c|c|c|c|c|}
\hline Mod. & \multicolumn{3}{|c|}{ HEC-RAS } & \multicolumn{3}{c|}{ Iber } & \multicolumn{3}{c|}{ Telemac-Mascaret } \\
\hline S/V & $\bar{U}$ & $\bar{u}$ & $\bar{v}$ & $\bar{U}$ & $\bar{u}$ & $\bar{v}$ & $\bar{U}$ & $\bar{u}$ & $\bar{v}$ \\
\hline S1 & 0.101 & 0.085 & -0.896 & 0.023 & 0.023 & 0.002 & 0.378 & 0.378 & 0.292 \\
\hline S2 & 0.925 & 0.899 & 0.467 & 0.959 & 0.959 & 0.935 & 0.938 & 0.938 & 0.920 \\
\hline S3 & 0.995 & 0.995 & 0.985 & 0.992 & 0.992 & 0.992 & 0.995 & 0.995 & 0.995 \\
\hline S4 & 0.950 & 0.802 & 0.103 & 0.981 & 0.981 & 0.976 & 0.985 & 0.985 & 0.952 \\
\hline S5 & 0.822 & 0.820 & 0.660 & 0.819 & 0.819 & 0.818 & 0.890 & 0.890 & 0.889 \\
\hline S6 & 0.822 & 0.815 & 0.878 & 0.965 & 0.965 & 0.964 & 0.920 & 0.920 & 0.918 \\
\hline S7 & 0.832 & 0.829 & 0.581 & 0.979 & 0.979 & 0.978 & 0.942 & 0.942 & 0.941 \\
\hline S8 & 0.791 & 0.791 & 0.478 & 0.873 & 0.873 & 0.874 & 0.917 & 0.917 & 0.917 \\
\hline
\end{tabular}


La comparación numérico-experimental es aceptable si el coeficiente de correlación tiende a $10-1$, y en la sección S5 es menor que 0.9; en la margen izquierda de la sección S5 en las pruebas del laboratorio se observó una zona de recirculación intermitente; se determinó que corresponde a una zona de separación del flujo y de inicio de remolinos que se propagan aguas abajo, por lo tanto, una razón para la baja correlación.

Por otra parte, la forma en planta del canal (curvatura compuesta y ancho variable) provoca un comportamiento particular del flujo, propio de ríos meandriformes. Así, la disminución del ancho del canal de $0.78 \mathrm{~m}$ (S1) a 0.40 m (S3) por la presencia de la curva cóncava-convexa en la margen izquierda del canal primero genera la disminución del componente de la velocidad $\bar{u}$ (Figura $3 a$ ) y velocidades negativas en el componente $\bar{v}$ (Figura $3 \mathrm{~b}$ ) en las inmediaciones de la sección S2. En la sección S3 el flujo se acelera y la velocidad en el componente $\bar{u}$ aumenta; se mantiene hasta la sección S7 (Figura 3a). La curva convexa-cóncava que permite la expansión del ancho del canal de 0.40 a $0.50 \mathrm{~m}$ en la margen izquierda (S3 a S7) presenta una expansión no simétrica que ocasiona un aumento del componente de la velocidad $\bar{v}$ (Figura $3 \mathrm{~b}$ ) y una disminución del componente de la velocidad $\bar{u}$ (Figura $3 a$ ), esto es, un gradiente de velocidades entre las secciones S5 a S7 (Figura 4b), comportamiento que los modelos empleados no pueden reproducir.

Profundizando en la misma dirección, el gradiente de velocidades (Figura 4b) genera el desarrollo de una capa de corte (velocidades menores que las del flujo principal), indicada por una delgada curva en la 
Figura 4b; el flujo entre la pared izquierda y la capa de corte es altamente turbulento y presenta velocidades bajas, comparadas con las velocidades del flujo principal (Figura 4a) y separación de flujo (color rojo en la Figura 4b); estos procesos hidráulicos son analizados en este trabajo en 2D, pero en esencia son en $3 \mathrm{D}$, por lo que las fluctuaciones de la velocidad ( $u^{\prime}$ y $v^{\prime}$ ) aumentan y, por lo tanto, se ve reflejado en la energía cinética de la turbulencia ( $k$ ) (Figura 6), principalmente en la margen izquierda entre las secciones S5-S7; zona en donde los valores de la velocidad calculados difieren de los valores medidos.

Finalmente, el comportamiento del número de Reynolds, que permite establecer y comprender la importancia de la relación de las fuerzas de inercia (aceleración) y las fuerzas viscosas (fricción) (Çengel \& Cimbala, 2010), muestra la aceleración del flujo al considerar que las variaciones en las fuerzas viscosas son pequeñas, ello también indica que los efectos de la rugosidad de la pared son despreciables.

\section{Conclusiones y recomendaciones}


En este trabajo se realizó la comparación numérico-experimental de los componentes de la velocidad y su magnitud en una canal rectangular con curvatura horizontal compuesta y los calculados con tres sistemas de modelación matemática bidimensional de uso libre más empleados en la práctica en la ingeniería de ríos para el cálculo de la hidrodinámica y la turbulencia.

Del análisis de errores realizado de los 45 puntos de medición se determina que los tres modelos tienen un comportamiento similar y aceptable, con una aproximación promedio de las tres variables de velocidad superior al $93 \%$, siendo Telemac-Mascaret (94.9\%) el que se aproxima ligeramente mejor, después Iber (93.7 \%) y finalmente HECRAS (92.4\%) en este trabajo. Sin embargo, al evaluar las variables de la velocidad por sección, la aproximación en el componente $\bar{v}$ con HEC-RAS disminuye hasta $40.7 \%$, mientras que Iber presenta una aproximación del $81.7 \%$ y Telemac-Mascaret del $85.3 \%$.

Al observar los campos de los componentes de velocidad se determina que $\bar{u}$ aumenta poco antes de la sección S3, no se presenta de manera uniforme a lo ancho de la sección y se concentra principalmente en la margen izquierda, después el núcleo de máxima velocidad pasa de la MI a la MD (secciones S3 a S5); para el componente $\bar{v}$, la velocidad máxima se concentra cerca de la MI en la sección S5, y la mínima en la misma margen entre las secciones S2 y S3.

El número de Reynolds indica un flujo turbulento. Si se considera que la variación de la longitud característica es similar en todo el canal y la variación de la viscosidad es pequeña permite observar la aceleración 
Tecnología y

Ciencias $\stackrel{\Xi}{\Im}$ Aua
2022, Instituto Mexicano de Tecnología del Agua

Open Access bajo la licencia CC BY-NC-SA 4.0

(https://creativecommons.org/licenses/by-nc-sa/4.0/)

del flujo. Así se identifica una zona cerca de la margen izquierda en donde la relación es menor, es decir, el flujo se desacelera sobre la margen izquierda entre las secciones S4 a la S8. También se determina el desarrollo de la capa de corte con el gradiente de velocidades del flujo (Figura 4b). Esta capa afecta la distribución del flujo después de la sección S4, ocasionando que el flujo principal se desvíe hacia el centro y a la MD del canal, concentrando el núcleo de velocidad máxima en esta margen. La zona entre la MI y la capa de corte es una zona de separación de flujo, producto de la expansión gradual en el ancho del canal, lo que permite el desarrollo de flujos secundarios, ya que al disminuir la velocidad $\bar{u}, y$ aunque el número de Reynolds disminuye dentro de esta zona, la energía cinética de la turbulencia ( $k$ ) aumenta; con base en lo anterior, una de las conclusiones importantes es que esta zona no puede ser reproducida por los modelos numéricos basados en la hipótesis de Saint Venant, y esto lo demuestra la comparación numérico-experimental de las velocidades mostradas en la Figura 2 , en los puntos localizados cerca de la MI entre las secciones S5-S8.

En general, la hidrodinámica del canal con la geometría presentada - calculada por los modelos numéricos empleados - tiene en promedio una aproximación superior al 93 \%, siguiendo la hipótes is de que el campo de velocidades localizado a 0.6 de la profundidad del flujo, que para fines de aplicación ingenieril resulta aceptable. La zona de menor aproximación (margen izquierda de las secciones S4-S7) pone en duda la hipótesis 2DH, dado que es mucho más compleja de reproducir y el modelo numérico no es el adecuado. Sin embargo, este fenómeno es importante, pues la expansión puede afectar de forma considerable la redistribución del flujo, 
sobre todo en estructuras hidráulicas, que es en donde se debe tener un control adecuado del flujo, por lo que el análisis presentado debe ser considerado en el diseño. Por su parte, la aplicación de este análisis en ríos meandriformes también plantea una línea de investigación debido a los efectos en la migración de las márgenes al recibir la incidencia del flujo en ellas, al igual que el fenómeno de transporte de sedimentos, y la interacción de la evolución del fondo y la adaptación del flujo, lo que sin duda modifica su comportamiento.

Finalmente, se recomienda ampliar las mediciones con un mayor número de puntos, medir a distintas profundidades y ampliar a la zona que no es reproducible en este trabajo, con la finalidad de mejorar el conocimiento del comportamiento del flujo en canales curvos; esto, con el objetivo de adentrarse en las líneas de investigación planteadas.

\section{Agradecimientos}

Los autores agradecen el apoyo de la Dirección General de Cómputo y de Tecnologías de Información y Comunicación (DGTIC) de la Universidad Nacional Autónoma de México (UNAM), por los recursos de supercómputo para el desarrollo de los cálculos numéricos asignados bajo el proyecto LANDCAD-UNAM-DGTIC-389, y al Departamento de Sistemas Linux y Súper Cómputo de la Secretaría de Telecomunicaciones e Informática del Instituto de Ingeniería de la UNAM. 
Tecnología y

Ciencias $\stackrel{\Xi}{\Im}$ Aua
2022, Instituto Mexicano de Tecnología del Agua

Open Access bajo la licencia CC BY-NC-SA 4.0

(https://creativecommons.org/licenses/by-nc-sa/4.0/)

\section{Referencias}

Abad, J. D., Frias, C. E., Buscaglia, G. C., \& Garcia, M. H. (2013). Modulation of the flow structure by progressive bedforms in the Kinoshita meandering channel. Earth Surface Processes and Landforms, 38(13), 1612-1622. DOI: 10.1002/esp.3460

Abad, J. D., \& Garcia, M. H. (2006). Hydrodynamics in Kinoshitagenerated meandering bends: Importance for river-planform evolution. River, Coastal and Estuarine Morphodynamics: RCEM 2005-Proceedings of the 4th IAHR Symposium on River, Coastal and Estuarine Morphodynamics, Urbana, USA, 2(1), 761-771.

Abad, J. D., \& Garcia, M. H. (2009). Experiments in a high-amplitude Kinoshita meandering channel: 1 . Implications of bend orientation on mean and turbulent flow structure. Water Resources Research, 45(2). DOI: $10.1029 / 2008$ WR007016.

Anderson, S., \& Lohrmann, A. (1995). Open water test of the SonTek acoustic Dopplervelocimeter. St. Petersburg, FL, USA: Proceedings of the IEEE Fifth Working Conference on Current Measurement, 188192. DOI: $10.1109 / \mathrm{ccm} .1995 .516172$

Bladé, E., Cea, L., \& Corestein, G. (2014a). Modelización numérica de inundaciones fluviales. Ingeniería del Agua, 18(1), 71-82.

Bladé, E., Cea, L., Corestein, G., Escolano, E., Puertas, J., VászquezCendón, M. E., Dolz, J., \& Coll, A. (2014b). Iber: herramienta de simulación numérica del flujo en ríos. Revista Internacional de Métodos Numéricos para cálculo y diseño en en Ingeniería, 30(1), 1- 
10. DOI: $10.1016 /$ j.rimni.2012.07.004

Bombar, G., Elçi, Ş., Tayfur, G., Güney, M. Ş., \& Bor, A. (2011). Experimental and numerical investigation of bed-load transport under unsteady flows. Journal of Hydraulic Engineering, 137(10), 12761282. DOI: $10.1061 /($ asce)hy.1943-7900.0000412

Cea, L., Puertas, J., \& Vázquez-Cendón, M. E. (2007). Depth averaged modelling of turbulent shallow water flow with wet-dry fronts. Archives of Computational Methods in Engineering, 14(3), 303-341. DOI:10.1007/s11831-007-9009-3

Çengel, Y. A., \& Cimbala, J. M. (2010). Fluid mechanics. Fundamentals and applicactions. New York, USA: McGrawHill.

CHC, Canadian Hydraulics Centre. (2011). Blue Kenue Reference manual. Ontario, Canada: Canadian Hydraulics Centre, National Research Council.

CIMNE, Centre Internacional de Mètodes Numèrics a I'Enginyeria . (2009). GiD. The universal, adaptative and user friendly pre and postprocessing system for computer analysis in science and engineering. Reference manual. Recuperado de http://www.gidhome.com

Da-Silva, A. M. F., El-Tahawy, T., \& Tape, W. D. (2006). Variation of flow pattern with sinuosity in sine-generated meandering streams. Journal of Hydraulic Engineering, 132(10), 1003-1014. DOI: 10.1061/(asce)0733-9429(2006)132:10(1003)

EDF-R\&D. (2016). Telemac modelling system. 3D hydrodynamics. Operating manual (release 7.1). Francia: EDF-R\&D. 
Tecnología y

Ciencias Agua
2022, Instituto Mexicano de Tecnología del Agua

Open Access bajo la licencia CC BY-NC-SA 4.0

(https://creativecommons.org/licenses/by-nc-sa/4.0/)

Fennema, R. J., \& Chaudhry, M. H. (1990). Explicit Methods for 2-D transient free surface flows. Journal of Hydraulic Engineering, 116(8), 1013-1034. DOI: 10.1061/(ASCE)0733-9429(1990)116:8(1013)

García, D. A., \& Matamoros, H. (2019). Análisis y comparación de los resultados de la simulación de flujo de un modelo físico a escala con el modelo numérico utilizado por el software IBER 2D. Revista de la Escuela Colombiana de Ingeniería, 114(2019), 77-85.

Garcia, R., \& Kahawita, R. A. (1986). Numerical solution of the St. Venant equations with MacCormack finite-difference Scheme. International Journal for Numerical Methods in Fluids, 6(5), 259-274. DOI: 10.1002/fld. 1650060502

Ghamry, H. K., \& Steffler, P. M. (2005). Two-dimensional depth-averaged modeling of flow in curved open channels. Journal of Hydraulic Research, 43(1), 44-55. DOI: 10.1080/00221680509500110

Gropp, W., Lusk, E., \& Skijellum, A. (2014). Using MPI: Portable parallel programming with the message-passing interface ( $3^{\text {rd }}$ ed.). Cambridge, USA: The MIT Press.

Guo, J. (2017). Exact procedure for Einstein-Johnson's sidewall correction in open channel flow. Journal Hydraulic Engineering, 143(4), 06016027. DOI: 10.1061/(asce)hy.1943-7900.0001260

Hafnaoui, M. A., \& Debabeche, M. (2020). Numerical modeling of the hydraulic jump location using 2D Iber software. Modeling Earth Systems and Environment. DOI: 10.1007/s40808-020-00942-3

HEC-RAS. (2016a). 2D Modelling user's manual. River Analysis System (version 5.0). Davis, USA: Hydrologic Engineering Center, Institute 
for Water Resources, U. S. Army Corps of Engineers.

HEC-RAS. (2016b). Hydraulic reference manual. River Analysis System (version 5.0). Davis, USA: Hydrologic Engineering Center, Institute for Water Resources, U. S. Army Corps of Engineers.

HEC-RAS. (2016c). User's manual. River Analysis System (version 5.0). Davis, USA: Hydrologic Engineering Center, Institute for Water Resources, U. S. Army Corps of Engineers.

Hervouet, J. M. (2007). Hydrodynamics of free surface flows: Modelling with the finite element method. Chichester, England: John Wiley and Sons, Ltd. DOI: 10.1002/9780470319628

Horritt, M. S., Di-Baldassarre, G., Bates, P. D., \& Brath, A. (2007). Comparing the performance of a 2-D finite element and a 2-D finite volume model of floodplain inundation using airborne SAR imagery. Hydrological Processes, 21(20), 2745-2759. DOI: 10.1002/hyp.6486

Jiménez, A. A., \& Berezowsky, M. (2004). Modelación del flujo bidimensional horizontal con coordenadas curvilíneas generales (SID 641). Ciudad de México, México: Instituto de Ingeniería, Universidad Nacional Autónoma de México.

Kalkwijk, J. P. T., \& De-Vriend, H. J. (1980). Computation of the flow in shallow river bends. Journal of Hydraulic Research, 18(4), 327-342. DOI: $10.1080 / 00221688009499539$

Karypis, G. (2013). METIS. A software package for partitioning unstructured graphs and computing fill-reduced orderings of sparse matrices (version 5.1.0.). Minneapolis, USA: Department of Computer Science \& Engineering, Univesity of Minnesota. 
Kaveh, K., Reisenbüchler, M., Lamichhane, S., Liepert, T., Nguyen, N. D., Bui, M. D., \& Rutschmann, P. (2019). A comparative study of comprehensive modeling systems for sediment transport in a curved open channel. Water (Switzerland), 11(9). DOI: 10.3390/w11091779

Knight, D. W. (2014). River hydraulics - A view from midstream. Journal of Hydraulic Research, 52(1), 138-139. DOI: $10.1080 / 00221686.2013 .855270$

Kraus, N. C., Lohrmann, A., \& Cabrera, R. (1994). New acoustic meter for measuring 3D laboratory flows. Journal of Hydraulic Engineering, 120(3), 406-412. DOI: 10.1061/(ASCE)0733-9429(1994)120:3(406)

Lane, S. N., Biron, P. M., Bradbrook, K. F., Butler, J. B., Chandler, J. H., Crowell, M. D., McLelland, S. J., Richards, K. S., \& Roy, A. G. (1998). Three-dimensional measurement of river channel flow processes using acoustic Doppler velocimetry. Earth Surface Processes and Landforms, 23(13), 1247-1267. DOI: 10.1002/(SICI)10969837(199812)23:13<1247::AID-ESP930>3.0.CO;2-D

Lohrmann, A., Cabrera, R., \& Kraus, N. C. (1994). Acoustic-Doppler velocimeter (ADV) for laboratory use. Proceedings of the Symposium on Fundamentals and Advancements in Hydraulic Measurements and Experimentation (pp. 351-365), ASCE, Buffalo, USA.

López, F., \& Garcia, M. H. (2001). Mean flow and turbulence structure of open-channel flow through non-emergent vegetation. Journal of Hydraulic Engineering, 127(5), 392-402. DOI: 10.1061/(ASCE)07339429(2001)127:5(392)

Luo, E. C. R. (2013). Hydrodynamic characteristics of expanded channels 
with their applications -the state-of-the-art. American Journal of Civil Engineering, 1(1), 31-40. DOI: 10.11648/j.ajce.20130101.15

McLelland, S. J., \& Nicholas, A. P. (2000). A new method for evaluating errors in high-frequency ADV measurements. Hydrological Processes, 14(2), 351-366. DOI: 10.1002/(sici)10991085(20000215)14:2<351::aid-hyp963>3.3.co;2-b

Menhenhall, W., Beaver, R. J., \& Beaver, B. M. (2010). Introducción a la probabilidad y estadística (13a ed.). México, DF, México: Cengage Learning.

Nikora, V., \& Goring, D. (1998). ADV measurements of turbulence: Can we improve their interpretation? Journal of Hydraulic Engineering, 124(6), 630-634. DOI: 10.1061/(ASCE)0733-9429(1998)124:6(630)

Pinos, J., \& Timbe, L. (2019). Performance assessment of twodimensional hydraulic models for generation of flood inundation maps in mountain river basins. Water Science and Engineering, 12(1), 1118. DOI: $10.1016 /$ j.wse.2019.03.001

Rastogi, A. K., \& Rodi, W. (1978). Predictions of heat and mass transfer in open channels. Journal Hydraulics Division, 104(3), 397-420.

Rousseau, Y. Y., Biron, P. M., \& Van-de-Wiel, M. J. (2016). Sensitivity of simulated flow fields and bathymetries in meandering channels to the choice of a morphodynamic model. Earth Surface Processes and Landforms, 41(9), 1169-1184. DOI: 10.1002/esp.3885

Rozovskii, I. L. (1957). Flow of water in bends of open channels. Kiev, Ukraine: Academy of Sciences of the Ukrainian SSR. 
Ruonan, B., Liekai, C., Xingkui, W., \& Danxun, L. (2016). Comparison of ADV and PIV measurements in open channel flows. Procedia Engineering (12th International Conference on Hydroinformatics (HIC 2016)-Smart Water for the Future), 154, 995-1001. DOI: 10.1016/j.proeng.2016.07.588

Shustikova, I., Domeneghetti, A., Neal, J. C., Bates, P., \& Castellarin, A. (2019). Comparing 2D capabilities of HEC-RAS and LISFLOOD-FP on complex topography. Hydrological Sciences Journal, 64(14), 17691782. DOI: $10.1080 / 02626667.2019 .1671982$

Song, C. G., Seo, I. W. , \& Kim, Y. D. (2012). Analysis of secondary current effect in the modeling of shallow flow in open channels. Advances in Water Resources, 41(2012), 29-48. DOI: 10.1016/j.advwatres.2012.02.003

SonTek. (1997). Pulse coherent Doppler processing and the ADV correlation (November). San Diego, USA: SonTek Technical Note.

Termini, D. (2009). Experimental observations of flow and bed processes in large-amplitude meandering flume. Journal of Hydraulic Engineering, 135(7), 575-587. DOI: 10.1061/(asce)hy.19437900.0000046

Voulgaris, G., \& Trowbridge, J. H. (1998). Evaluation of the acoustic doppler velocimeter (ADV) for turbulence measurements. Journal of Atmospheric and Oceanic Technology, 15(1), 272-289. DOI: 10.1175/1520-0426(1998)015<0272:eotadv>2.0.co;2

Wahl, T. L. (2000). Analyzing ADV data using WinADV. Joint Conference on Water Resource Engineering and Water Resources Planning and 
Management, Minneapolis, USA. DOI: 10.1061/40517(2000)300

Wu, W., Wang, P., \& Chiba, N. (2004). Comparison of five depth-averaged 2-D turbulence models for river flows. Archives of Hydro-Engineering and Environmental Mechanics, 51(2), 183-200. 